\title{
Extension of the Misme and Fimbel Model for the Estimation of the Cumulative Distribution Function of the Differential Rain Attenuation Between Two Converging Terrestrial Links
}

\author{
Henrique Grynszpan, Emanoel Costa, \\ Centro de Estudos em Telecomunicações (CETUC) \\ Pontifícia Universidade Católica do Rio de Janeiro (PUC-Rio) \\ Rua Marquês de São Vicente 225, 22451-900 Rio de Janeiro RJ, BRAZIL \\ email: epoc@cetuc.puc-rio.br
}

\begin{abstract}
A distribution-free model is presented for the cumulative distribution function of the differential rain attenuation between two co-channel converging terrestrial links operating at frequencies above $10 \mathrm{GHz}$. This is accomplished through an extension of the Misme and Fimbel model, which determines the cumulative distribution of the rain attenuation on an isolated link from its parameters and data for the radio climatic region, as well as concepts from probability theory. Next, model predictions and experimental results are compared and effects from variations of the angle between the links and of the path length are studied. Finally, rain effects on the cumulative distribution of the $\mathrm{C} / \mathrm{I}$ ratio between the desired and interference powers at the same receiver are analyzed, considering the angular discrimination of the receiving antenna.
\end{abstract}

Index Terms-Converging links, Differential rain attenuation, Interference between terrestrial radio links.

\section{INTRODUCTION}

Frequency bands above $10 \mathrm{GHz}$ have been increasingly used in point-to-point, high-bandwidth, wireless access of clients to local- and wide-area networks. The access point generally adopts a star topology, with many wireless terrestrial links converging to a common central station. It is well known that rain is the dominant propagation impairment factor to links operating in these frequency bands and that the design of such star networks should consider the possibility that a link can be severely attenuated by rain while experiencing interference by another link operating at the same frequency but under milder conditions. In other words, the design of the network should consider the effects from differential rain attenuation on link availability. It was observed that some rain unavailability calculations for these networks were conservatively performed, leading to a relatively inefficient use of the frequency spectrum. These calculations only considered rain attenuation in the desired link, assuming clear-sky conditions for the interfering link, making it necessary to increase the angle between them. A more realistic consideration would be that rain cells could simultaneously attenuate both links, allowing a decrease of the angle between the links and an increase in spectral 
efficiency. Indeed, efficiency is achieved if the central station serves a large number of remote terminals and if the same frequency is reused as often as possible by the converging links.

Frequency-sharing studies in converging links are generally based on models for the cumulative distribution function of differential rain attenuation, some of which are available in the literature. For example, Morita et al. [1]-[4] developed a model based on the Gamma distribution for the joint probability density function of the attenuations on two links, assumed to be correlated. The parameters of the distribution were determined from those of another Gamma distribution, adjusted to the measured cumulative distribution function of the point rainfall rate, in combination with an assumed spatial structure for rain field. The cumulative distribution function of differential rain attenuation between the links was numerically determined. Model predictions were compared with results from measurements performed by the same authors on three short links operating at frequencies in the 19-GHz band, converging at the Musashino Electrical Communication Laboratory (ECL), Japan. The model proposed by Kanellopoulos et al. [5],[6] differs from the one by Morita et al. [1]-[4] by the assumptions of Lognormal distributions for the cumulative distributions of the rainfall rate, rain attenuation on an individual link, as well as for the joint probability density function of the attenuations on two links. Model predictions were compared with results from measurements performed by the Radio Propagation Group at the Centro de Estudos em Telecomunicações da Pontifícia Universidade Católica do Rio de Janeiro (CETUC/PUC-Rio) on links operated by Empresa Brasileira de Telecomunicações (Embratel) at frequencies in the $15-\mathrm{GHz}$ band, converging at the Rua dos Ingleses station (RIS), City of São Paulo, Brazil [7],[8]. It is interesting to observe that there are authors who advocate the application of the Gamma and the Lognormal distributions to model rainderived statistics resulting from data obtained in tropical and temperate areas, respectively. Indeed, Andrade at al. [9] concluded that the Inverse Gaussian and Gamma distributions were the most adequate statistical models for the rain attenuation in the terrestrial links converging at the RIS station (in a tropical region), for medium and high attenuation levels. Recently, Paulson et al. [10] adopted the Lognormal law for joint probability density function of the attenuations on two links. However, the parameters of the distribution were determined from measurements performed by the Chibolton meteorological radar, United Kingdom. The model was used to determine diversity improvement (DI) and diversity gain (DG) factors for converging links, using a maximum likelihood estimation method. While the above could be classified as parametric methods, the empirical model by Pérez García et al. [8] was based on measurements of the cumulative distribution functions of differential rain attenuation on several pairs of links and of rain attenuation on individual links. These measurements were performed at the already mentioned Rua dos Ingleses station (four $15-\mathrm{GHz}$ links and two 18GHz links), as well as in the City of Brasília, Brazil (seven 23-GHz links and six 38-GHz links). Prediction is based on an analytic expression with coefficients determined by curve fitting to experimental data. This expression uses the operating frequency, the angle between the two links, as 
well as the difference in path lengths to map the cumulative distribution functions of rain attenuation on the two individual links into that of the differential rain attenuation.

The present work will present a distribution-free model based on fundamental principles and on the measured cumulative distribution function of point rainfall rate (without assuming any specific probability law) to determine the cumulative distribution function of the differential rain attenuation between two converging links operating at the same frequency, greater than $10 \mathrm{GHz}$. The development extends the Misme and Fimbel model [11], which determines the cumulative distribution function of rain attenuation on an isolated link from its parameters and radio climatic data for the region. Thus, the present model at least initially follows a different approach from those leading to the previous methods. Next, the model numerically determines the joint cumulative distribution function of the attenuations on the two links over a fine grid of two-dimensional joint attenuation points. From this partial result, the cumulative distribution function of the differential rain attenuation between the links is easily determined by a simple summation. The present model generalizes the development by Stola [12], also based on the Misme and Fimbel procedures but limited to the determination of the cumulative distribution function of the rain attenuation simultaneously exceeded on two converging links. Following this Introduction, Section II will briefly review the original Misme and Fimbel model [11] and will discuss its extension to the determination of the cumulative distribution function of the differential rain attenuation between two co-channel converging links. Section III will describe experimental campaigns providing input data for comparison with model predictions and present effective values for climatic parameters. Section IV will compare calculation and measurement results. Additionally, it will present model predictions of the effects of variations of the angle between the links and the path lengths on the cumulative distribution function of the differential rain attenuation. The features of the present model make it possible to provide explanations for the observed changes of the cumulative distribution function due to variation on these parameters. Based on the extended Misme and Fimbel model, Section V will analyze the effects of the differential rain attenuation on the availability of co-channel converging links according to the conservative and more realistic procedures, considering the angular discrimination of the receiving antenna. Finally, conclusions will be presented in Section VI.

\section{THE MISME AND FIMBEL MODEL AND ITS EXTENSION}

The Misme and Fimbel model [11] for the estimation of the cumulative distribution function of the rain attenuation on an isolated terrestrial link adopts the following simplifying assumptions for the rain field:

- intense precipitation occurs in the form of circular cylindrical cells with constant rainfall rate $R$ $(\mathrm{mm} / \mathrm{h})$;

- the average diameter $d(R)(\mathrm{km})$ of a rain cell can be related to the corresponding rainfall rate $R$ through the expression 


$$
\mathrm{d}(\mathrm{R})=\mathrm{d}_{\mathrm{o}}(100 / \mathrm{R})^{\beta}
$$

with the parameter values $d_{o}=2.2 \mathrm{~km}$ and $\beta=0.4$, as long as $d(R) \leq d_{\max }$ (the maximum cell diameter, equal to $33 \mathrm{~km}$ );

- the point rainfall rate is a random variable characterized by its measured cumulative distribution function $P_{r}(R)$ that is independent from the observation point within the region of interest;

- at each instant of time, attenuation on a link is caused by a single rain cell;

- the specific attenuation $\gamma(\mathrm{dB} / \mathrm{km})$ can be related to the rainfall rate $R(\mathrm{~mm} / \mathrm{h})$ through the expression $\gamma=k R^{\alpha}$, with the parameters $k$ and $\alpha$ being determined as functions of the operating frequency and the polarization according to the most recent version of Recommendation ITU-R P.838 [13].

It should be noted that, to simplify numerical procedures, instead of explicitly considering the small contribution from residual precipitation to rain attenuation according to the Misme and Fimbel model specification [10], these effects have been included by extending the validity of expression (1) to rainfall rates below the original threshold of $10 \mathrm{~mm} / \mathrm{h}$. Even though relevant developments based on the Misme and Fimbel model have been proposed with the objectives of simplifying or improving the corresponding algorithms [14], the present work will strictly adhere to all the characteristics of the original model, except for the slight modification described above.

As illustrate din Figure 1, the intersection $L_{o l}(\mathrm{~km})$ between a cell with rainfall rate $R$ and link $A B$ with path length $D_{l}(\mathrm{~km})$ causes the attenuation $A_{o l}=k R^{\alpha} L_{o l}(\mathrm{~dB})$. When this rain cell is displaced by all possible manners while keeping the length $L_{o l}$ of the intersection fixed, the cell center describes the dashed geometric locus $V_{11} V_{21} I_{1} V_{31} V_{41} I_{2}$ also shown in Figure 1. The geometric locus limits the area $S\left(A_{o l}, R\right)$ defined by [11]

$$
\mathrm{S}\left(\mathrm{A}_{\mathrm{o} 1}, \mathrm{R}\right)=\left(\mathrm{D}_{1}-\mathrm{L}_{\mathrm{o} 1}\right) \sqrt{\mathrm{d}^{2}-\mathrm{L}_{\mathrm{o} 1}^{2}}+\frac{1}{2}\left[\mathrm{~d}^{2} \arctan \left(\frac{\sqrt{\mathrm{d}^{2}-\mathrm{L}_{\mathrm{o} 1}^{2}}}{\mathrm{~L}_{\mathrm{o} 1}}\right)-\mathrm{L}_{\mathrm{o} 1} \sqrt{\mathrm{d}^{2}-\mathrm{L}_{\mathrm{o} 1}^{2}}\right]
$$

The two terms separated by the addition sign in the right-hand side of expression (2) represent the areas of the rectangle $V_{11} V_{21} V_{31} V_{41}$ and the two circular segments limited by the geometric locus associated with link $A B$, respectively. For each assumed value for $A_{o l}$, a minimum rainfall rate $R_{\min }$ $(\mathrm{mm} / \mathrm{h})$ should be found to guarantee the inequalities

$$
\mathrm{L}_{\mathrm{o} 1}\left(\mathrm{~A}_{\mathrm{o} 1}, \mathrm{R}_{\min }\right)=\mathrm{A}_{\mathrm{o} 1} /\left(\mathrm{kR}_{\min }^{\propto}\right) \leq \mathrm{d}\left(\mathrm{R}_{\min }\right) \quad \text { and } \quad \mathrm{L}_{\mathrm{o} 1}\left(\mathrm{~A}_{\mathrm{o} 1}, \mathrm{R}_{\min }\right)=\mathrm{A}_{\mathrm{o} 1} /\left(\mathrm{kR}_{\min }^{\propto}\right) \leq \mathrm{D}_{1}
$$

Its value should be determined from these inequalities and expression (1), considering the upper limit $d_{\max }$. It should be observed that the condition $R \geq R_{\min }$ keeps each term in the right-hand side of 
expression (2) real and positive or null. It is also noted that a cell with rainfall rate $R$ and center in the interior of the geometric locus will cause attenuation $A>A_{o l}$.

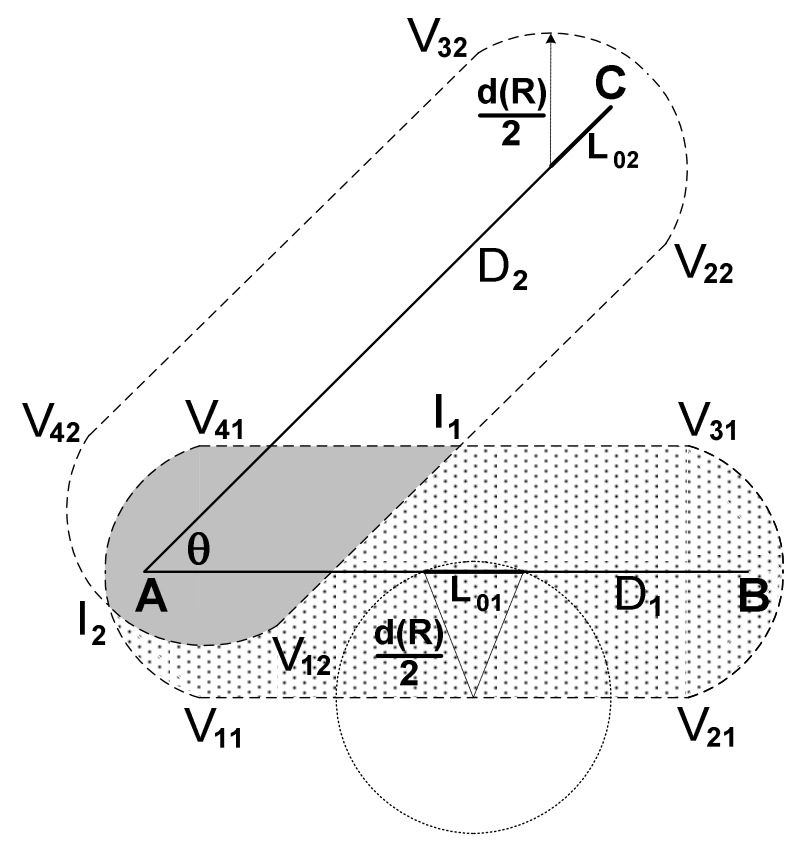

Fig. 1. Converging links $A B$ and $A C$ with respective path lengths $D_{1}$ and $D_{2}$ making the angle $\theta$ at the common terminal, associated geometric loci $\mathrm{V}_{11} \mathrm{~V}_{21} \mathrm{I}_{1} \mathrm{~V}_{31} \mathrm{~V}_{41} \mathrm{I}_{2}$ and $\mathrm{V}_{12} \mathrm{I}_{1} \mathrm{~V}_{22} \mathrm{~V}_{32} \mathrm{~V}_{42} \mathrm{I}_{2}$ corresponding to attenuations $\mathrm{A}_{\mathrm{o} 1}$ and $\mathrm{A}_{\mathrm{o} 2}$, respectively, and to a cylindrical cell of rainfall rate $R$, as well as the intersection $V_{12} I_{1} V_{41} I_{2}$ between the two geometric loci.

Based on the assumptions above, geometrical concepts and additional considerations, as well as on the total probability theorem [15], Misme and Fimbel established the following general expression for the cumulative distribution function of rain attenuation on the isolated link $A B$

$$
\operatorname{Pr}\left\{\mathrm{A}>\mathrm{A}_{\mathrm{o} 1}\right\}=\int_{\mathrm{R}_{\min }}^{\infty} \frac{\mathrm{S}\left(\mathrm{A}_{\mathrm{ol}}, \mathrm{R}\right)}{\pi \mathrm{d}^{2}(\mathrm{R}) / 4} \mathrm{p}_{\mathrm{r}}(\mathrm{R}) \mathrm{dR}=\int_{\mathrm{P}_{\min }}^{1} \frac{\mathrm{S}\left[\mathrm{A}_{\mathrm{ol}}, \mathrm{R}\left(\mathrm{P}_{\mathrm{r}}\right)\right]}{\pi \mathrm{d}^{2}\left[\mathrm{R}\left(\mathrm{P}_{\mathrm{r}}\right)\right] / 4} \mathrm{dP}
$$

In the original integral (reproduced between the equality signs in the above expression) [11], numerically evaluated for $A_{o l}$ values of interest, $p_{r}(R)$ is the probability density function of the point rainfall rate, assumed to follow a lognormal law with parameters determined from corresponding measurements. A trivial change of variable leads to the last integral of expression (4), which can be numerically evaluated using the measured cumulative distribution function of the point rainfall rate. That is, without resorting to particular probability distributions and also avoiding possible errors associated with numerical differentiations involved in the determination of $p_{r}(R)$ from $P_{r}(R)$.

Figure 1 also displays a second link $A C$ with path length $D_{2}(\mathrm{~km})$ that makes an angle $\theta$ (degrees) with the former at the common terminal $A$, as well as the associated geometric locus $V_{12} I_{1} V_{22} V_{32} V_{42} I_{2}$ corresponding to the same cell with rainfall rate $R$ and attenuation $A_{o 2}$. Based on the development summarized in the previous paragraphs, the joint probability that the attenuations in the two links simultaneously exceed the respective values $A_{o 1}$ and $A_{o 2}$ can be represented by 


$$
\operatorname{Pr}\left\{\mathrm{A}_{1}>\mathrm{A}_{\mathrm{o} 1}, \mathrm{~A}_{2}>\mathrm{A}_{\mathrm{o} 2}\right\}=\int_{\mathrm{P}_{\min }}^{1} \frac{\mathrm{S}_{\mathrm{n}}\left[\mathrm{A}_{\mathrm{o} 1}, \mathrm{~A}_{\mathrm{o} 2}, \mathrm{R}\left(\mathrm{P}_{\mathrm{r}}\right)\right]}{\pi \mathrm{d}^{2}\left[\mathrm{R}\left(\mathrm{P}_{\mathrm{r}}\right)\right] / 4} \mathrm{dP}_{\mathrm{r}}
$$

In the above expression, $S_{\cap}\left(A_{o l}, A_{o 2}, R\right)$ is the intersection between the surfaces limited by the two geometric loci, represented by the light gray area in Figure 1 , and $P_{\min }$ is the value of the rainfall rate cumulative distribution function corresponding to $R_{\min }=\max \left\{R_{\min 1}, R_{\min 2}\right\}$, where $R_{\min 1}$ and $R_{\min 2}$ are the minimum rainfall rates for the two individual links, respectively. Equation (5) generalizes the Misme and Fimbel model to consider two converging links and also extends the formulation presented by Stola [12], restricted to the particular case $A_{o l}=A_{o 2}$ and analyzed in more detail for $D_{l}=$ $D_{2}$. Once the configuration of interest has been specified, the integral in expression (5) can be numerically calculated for pairs of attenuation values $\left(A_{o l}, A_{o 2}\right)$ defined over a fine grid with resolution $\varepsilon$ (that is, $A_{o l}$ and $A_{o 2}$ assume the values $m \varepsilon$ and $n \varepsilon$, where $m, n=0,1, \ldots, N$, respectively).

The area $S_{\cap}\left(A_{o 1}, A_{o 2}, R\right)$ is exactly determined by means of an algorithm that combines simple computational geometry procedures [16]. It is observed that the geometric loci are convex polygons characterized by straight line or circular arc segments. Therefore, these features will be conserved by their intersection, defined by all: (i) vertices of one geometric locus located in the interior of the other ( $V_{12}$ and $V_{41}$ in Figure 1); and (ii) crossings between segments from different geometric loci $\left(I_{1}\right.$ and $I_{2}$ in Figure 1). Next, these points are sorted in the counter-clockwise sense and it is determined whether two consecutive points should be connected by a straight line or a circular arc segment. In the latter case, the position of the center of the corresponding circle is also determined. Finally, the intersecting polygon is subdivided into triangles and circular segments. The area $S_{\cap}\left(A_{o l}, A_{o 2}, \mathrm{R}\right)$ is exactly equal to the sum of the areas of these basic elements.

In principle, the cumulative distribution function of the differential rain attenuation $\operatorname{Pr}\left\{a_{1}-a_{2}>A_{o}\right\}$ would be determined by the double integration of the joint probability density function $\mathrm{p}_{\mathrm{a}_{1} \mathrm{a}_{2}}\left(\mathrm{~A}_{1}, \mathrm{~A}_{2}\right)$ in the region of interest, below the straight line $A_{1}-A_{2}=A_{o}$, as indicated by Figure 2 . That is,

$$
\operatorname{Pr}\left\{a_{1}-a_{2}>A_{o}\right\}=\int_{o}^{A_{\max }-A_{o}}\left[\int_{A_{2}+A_{o}}^{A_{\max }} p_{a_{1} a_{2}}\left(A_{1}, A_{2}\right) d A_{1}\right] d A_{2}
$$

where $A_{\max }$ is an upper limit beyond which the contribution from the above integral to the cumulative distribution function of the differential rain attenuation can be neglected. Tests have shown that the adopted value $A_{\max }=47 \mathrm{~dB}$ is adequate for this purpose. As indicated by expression (6), the integral between square brackets should be evaluated for an arbitrary value of $A_{2}$ in the interval $\left(0, A_{\text {max }}-A_{o}\right)$. The result, which is a function of $A_{2}$, should then be integrated in the same interval.

Figure 2 indicates that the region of interest can be approximately subdivided into a large number of narrow rectangles in such a way that 


$$
\begin{gathered}
\operatorname{Pr}\left\{a_{1}-a_{2}>A_{o}\right\} \approx \sum_{n=1}^{N} \int_{A_{2 n}-\varepsilon / 2}^{A_{2 n}+\varepsilon / 2} \int_{A_{2 n}+A_{o}}^{A_{\max }} p_{a_{1} a_{2}}\left(A_{1}, A_{2}\right) d A_{1} d A_{2} \approx \\
\approx \sum_{n=1}^{N} \operatorname{Pr}\left\{a_{1}>A_{2 n}+A_{o}, A_{2 n}-\varepsilon / 2<a_{2}<A_{2 n}+\varepsilon / 2\right\} \approx \\
\approx \sum_{n=1}^{N}\left[\operatorname{Pr}\left\{a_{1}>A_{2 n}+A_{o}, a_{2}>A_{2 n}-\varepsilon / 2\right\}-\operatorname{Pr}\left\{a_{1}>A_{2 n}+A_{o}, a_{2}>A_{2 n}+\varepsilon / 2\right\}\right]
\end{gathered}
$$

Note that the values for each term inside the square brackets in the last line of expression (7) have previously been calculated by equation (5) and stored. That is, $\operatorname{Pr}\left\{a_{1}-a_{2}>A_{o}\right\}$ values have been directly obtained from a summation of differences of $\operatorname{Pr}\left\{a_{1}>A_{o l}, a_{2}>A_{o 2}\right\}$ values, circumventing the time-consuming and numerically-unstable determination of $\mathrm{p}_{\mathrm{a}_{1} \mathrm{a}_{2}}\left(\mathrm{~A}_{1}, \mathrm{~A}_{2}\right)$.

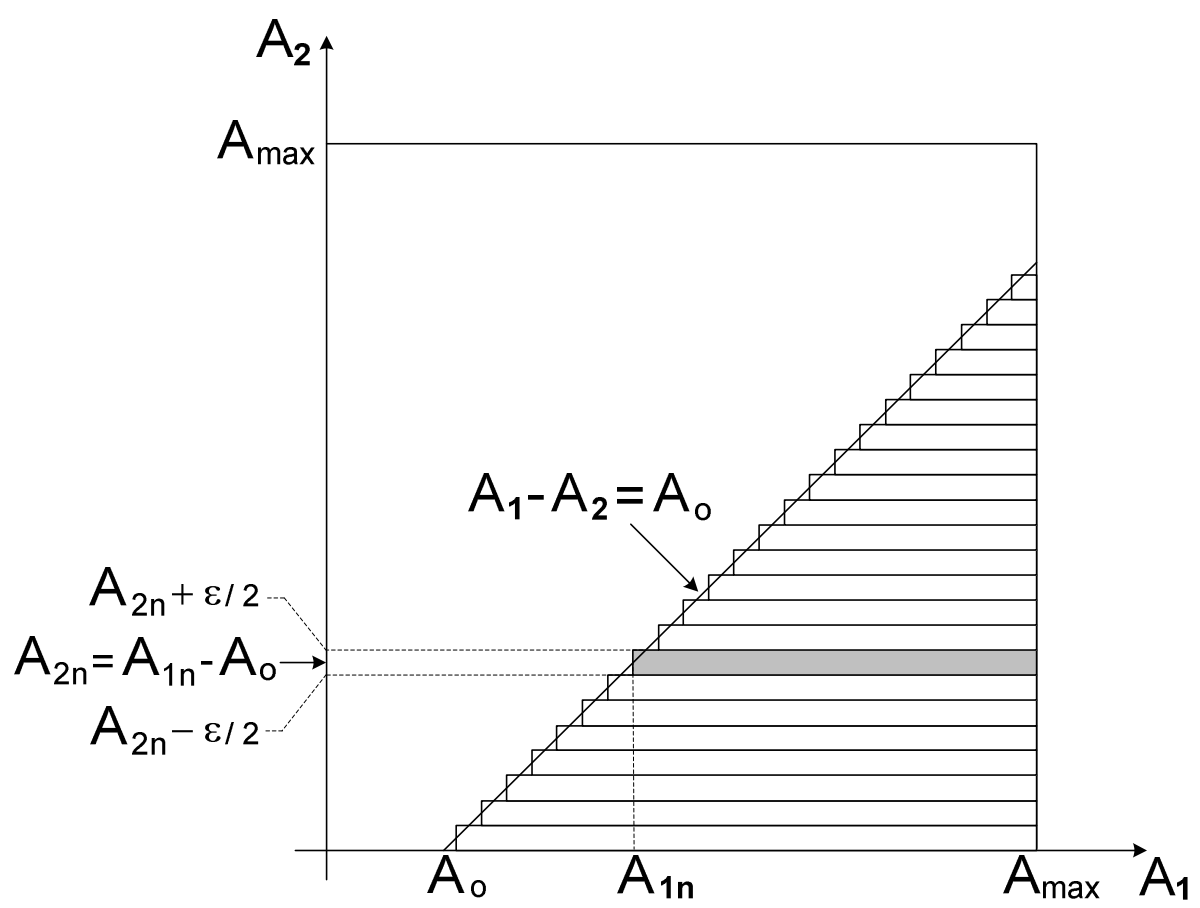

Fig. 2.Region of interest for the estimation of $\operatorname{Pr}\left\{a_{1}-a_{2}>A_{o}\right\}$ and its approximate subdivision into narrow rectangles.

Figure 2 also shows that the approximated region of integration represented by the narrow gray rectangle differs from the exact one by: (i) incorrectly considering a small triangle above the line $A_{1}$ $A_{2}=A_{o}$; and (ii) incorrectly neglecting another small triangle below same line. These triangles are images of each other around their common vertex and have the same area, which is small when compared to that of the gray rectangle. Additionally, the joint probability density function of the attenuations on the two links does not display discontinuities and the contributions of the two triangles are approximately equal. Therefore, the last line of equation (7) provides a very good approximation to $\operatorname{Pr}\left\{a_{1}-a_{2}>A_{o}\right\}$. Additionally, Figure 2 indicates that an upper bound to $\operatorname{Pr}\left\{a_{1}-a_{2}>A_{o}\right\}$ can be obtained by keeping the lower left vertices of the narrow rectangles at the line $A_{1}-A_{2}=A_{o}$ and that a lower bound can be obtained by keeping the upper left vertices of the narrow rectangles at the same 
line. Performed test have indicated that the two bounds converge very fast to each other and to the results from expression (7) as soon as the value of the differential attenuation $A_{o}$ exceeds a couple of decibels, for the assumed values for the increment $\varepsilon\left(0.01 \mathrm{~dB}\right.$ for $\mathrm{A}_{1}$ or $\mathrm{A}_{2}$ less than $1 \mathrm{~dB}$, since the joint probability displays fast variations in these regions, and $0.1 \mathrm{~dB}$ otherwise). It should be observed that the approach described by expression (7) could be used by any model that determines the cumulative distribution function of the differential rain attenuation from the joint cumulative distribution function of the attenuations on two links.

Finally, it is concluded from equations (5) and (7) that, for a set of link parameters, the extended Misme and Fimbel model maps the directly-measured cumulative distribution function of the rainfall rate onto that of the differential rain attenuation. Therefore, the ideal comparison between experimental results and model predictions should be based on cumulative distribution functions of the rainfall rate and the differential rain attenuation resulting from the same period of measurements. The results from such comparisons will be presented in the next section.

\section{INPUT DATA}

In a collection of papers published during the 1970's, Morita and co-workers [1]-[4] reported on measurements performed on three short $19-\mathrm{GHz}$ links converging at the Musashino Electrical Communication Laboratory (ECL), Japan. More recently [7],[8], similar measurements were performed in the City of São Paulo, Brazil, using the following links operated by Empresa Brasileira de Telecomunicações (Embratel) and converging at the central station of Rua dos Ingleses (RIS). The names of the remote stations, frequencies of operation, path lengths, polarizations (representing horizontal and vertical polarizations by $\mathrm{H}$ and $\mathrm{V}$, respectively) and azimuths (at ECL) of the links, as well as their periods of operation, are presented in Table I.

TABLE I. PARAMETERS AND PERIODS OF OPERATION OF THE LINKS

\begin{tabular}{|c|c|c|c|c|c|c|}
\hline Central Station & Remote Station & Frequency & Path Length & Polarization & Azimuth & Operation \\
\hline \multirow[t]{2}{*}{ ECL } & \multirow[t]{2}{*}{ Shakujii } & \multirow[t]{2}{*}{$19.1 \mathrm{GHz}$} & \multirow[t]{2}{*}{$4.3 \mathrm{~km}$} & \multirow[t]{2}{*}{$\mathrm{H}$} & \multirow[t]{2}{*}{$65.0^{\circ}$} & Jul.1971 to Sep.1971 \\
\hline & & & & & & Jan.1974 to Dec.1974 \\
\hline ECL & Shinkawa & $19.3 \mathrm{GHz}$ & $4.7 \mathrm{~km}$ & $\mathrm{H}$ & $180.0^{\circ}$ & Jan.1974 to Dec.1974 \\
\hline ECL & Sakai & $19.0 \mathrm{GHz}$ & $2.9 \mathrm{~km}$ & $\mathrm{H}$ & $228.0^{\circ}$ & Jul.1971 to Sep.1971 \\
\hline \multirow[t]{2}{*}{ RIS } & \multirow[t]{2}{*}{ Shell } & \multirow[t]{2}{*}{$18.6 \mathrm{GHz}$} & \multirow[t]{2}{*}{$7.5 \mathrm{~km}$} & \multirow[t]{2}{*}{$\mathrm{V}$} & \multirow[t]{2}{*}{$126.6^{\circ}$} & Aug.1994 to Mar.1995 \\
\hline & & & & & & Sep.1995 to Aug.1996 \\
\hline \multirow[t]{2}{*}{ RIS } & \multirow[t]{2}{*}{ Cenesp15 } & \multirow[t]{2}{*}{$14.6 \mathrm{GHz}$} & \multirow[t]{2}{*}{$12.8 \mathrm{~km}$} & \multirow[t]{2}{*}{$\mathrm{H}$} & \multirow[t]{2}{*}{$220.0^{\circ}$} & Apr.1994 to Mar.1995 \\
\hline & & & & & & Sep.1995 to Aug.1996 \\
\hline RIS & Cenesp18 & $18.6 \mathrm{GHz}$ & $12.8 \mathrm{~km}$ & $\mathrm{~V}$ & $220.0^{\circ}$ & Apr.1994 to Mar.1995 \\
\hline \multirow[t]{2}{*}{ RIS } & \multirow[t]{2}{*}{ Bradesco2 } & \multirow[t]{2}{*}{$14.6 \mathrm{GHz}$} & \multirow[t]{2}{*}{$12.8 \mathrm{~km}$} & \multirow[t]{2}{*}{$\mathrm{V}$} & \multirow[t]{2}{*}{$278.6^{\circ}$} & Apr.1994 to Dec.1994 \\
\hline & & & & & & Sep.1995 to Aug.1996 \\
\hline \multirow[t]{2}{*}{ RIS } & \multirow[t]{2}{*}{ Barueri } & \multirow[t]{2}{*}{$14.5 \mathrm{GHz}$} & \multirow[t]{2}{*}{$21.7 \mathrm{~km}$} & \multirow[t]{2}{*}{$\mathrm{V}$} & \multirow[t]{2}{*}{$290.4^{\circ}$} & Apr.1994 to Mar.1995 \\
\hline & & & & & & Sep.1995 to Aug.1996 \\
\hline
\end{tabular}

Angles between links can be determined from azimuth differences. In addition to received signal levels, the rainfall rate data were also acquired at ECL and RIS. The two data sets were processed, 
yielding cumulative distribution functions of one-minute point rainfall rate at the central station, attenuations on isolated links and differential attenuations on pairs of links.

Several authors performed rain cell size measurements using meteorological radars located in temperate regions [17]-[20], and a high-elevation slant path at a tropical site [21]. Some of the results were presented in the form of a functional relation between the average cell diameter and the rainfall rate and seem to be in reasonable agreement with the assumed values for parameters $\beta$ and $d_{o}$ of the model represented by expression (1). However, radar measurements performed in the Amazon region provided probabilities that a cell diameter be exceeded for rainfall rate thresholds in the convective regime that are substantially higher than those originating from temperate climates [22]. This may be taken as an indication that the original values are not universal. Moreover, it is easily verified that the combination of the assumption that attenuation on a link is due to a single rain cell with the originallyproposed values for $\beta$ and $d_{o}$, is not capable of explaining the measured attenuation values, which can reach $33 \mathrm{~dB}$ or more during $0.01 \%$ of the time for the RIS experiment.

Ideally, $d_{o}$ and $\beta$ should be determined from radar measurements, as described by items 2.2 and 2.3, as well as by Figure 1 of the original reference for the Misme and Fimbel model [11]. Note that these two parameters should depend only on the rain climate and should not depend on the parameters of any link, either operational or in the planning stage. Unfortunately, radar measurements of rain cell sizes are not available for all climatic regions of the world. To circumvent the absence of information, we described an alternative approximate method for the estimation of the two parameters, based on the cumulative distribution functions of the point rainfall rate and the rain attenuation on links simultaneously measured in the region of interest. To extend the applicability of the Misme and Fimbel model to the links converging at the RIS station, parameter values corresponding to the effective rain cell diameter were estimated by least-squares fitting a straight line $\left(y=\beta x+\log d_{o}\right)$ to data rearranged as indicated in the following equation

$$
y_{p}=\log \left[d\left(R_{p}\right)\right]=\log \left(\frac{A_{p}}{k R_{p}^{\alpha}}\right)=\beta \log \left(\frac{100}{R_{p}}\right)+\log d_{o}=\beta x_{p}+\log d_{o}
$$

where attenuation $A_{p}$ and rainfall rate $R_{p}$ values were obtained from the respective measured cumulative distributions for the same percentages of time $\mathrm{p}$. Specific attenuation values $\gamma=k R^{\alpha}$ were determined considering the appropriate polarizations and operating frequencies according to Recommendation ITU-R P.838-3 [13]. For reference purposes, the same calculations were also performed for links converging at the ECL station. Figure 3 presents the $\left(x_{p}, y_{p}\right)$ data sets corresponding to the links. The Figure also shows straight-line segments resulting from the least squares fit to data from each link and the estimated effective values of the parameters $\left(\log d_{o}\right)$ and $\beta$. As expected, the effective values for the parameter $d_{o}$ are larger than the original one for the relatively long RIS links. The present results are consistent with those from similar calculations performed by 
Sarkar et al. [23] using data from the Indian subcontinent. On the other hand, the effective $d_{o}$ values for the ECL links are closer to those estimated from radar measurements, in reasonable agreement with the original expression (1), and with the assumption that attenuation on shorter links would be mainly due to individual cells. Due to errors in the present alternative approximation method, the results in Figure 3 clearly indicate that the effective parameter values $d_{o}$ and $\beta$ derived from equation (8) depend on the path length, polarization and frequency of the links. However, to be consistent with the assumptions of the original Misme and Fimbel model [11], it will be assumed that $d_{o}$ and $\beta$ are exclusively climatic parameters. Therefore, the present work estimated the effective values $d_{o}=6.80$ $\mathrm{km}$ and $\beta=0.52$ for calculations involving the RIS links, and $d_{o}=2.80 \mathrm{~km}$ and $\beta=0.14$ for those corresponding to the ECL links, by the average values of the samples from the links. It should be remarked that tests indicated that the extended Misme and Fimbel model is relatively insensitive to variations in the parameter $\beta$.

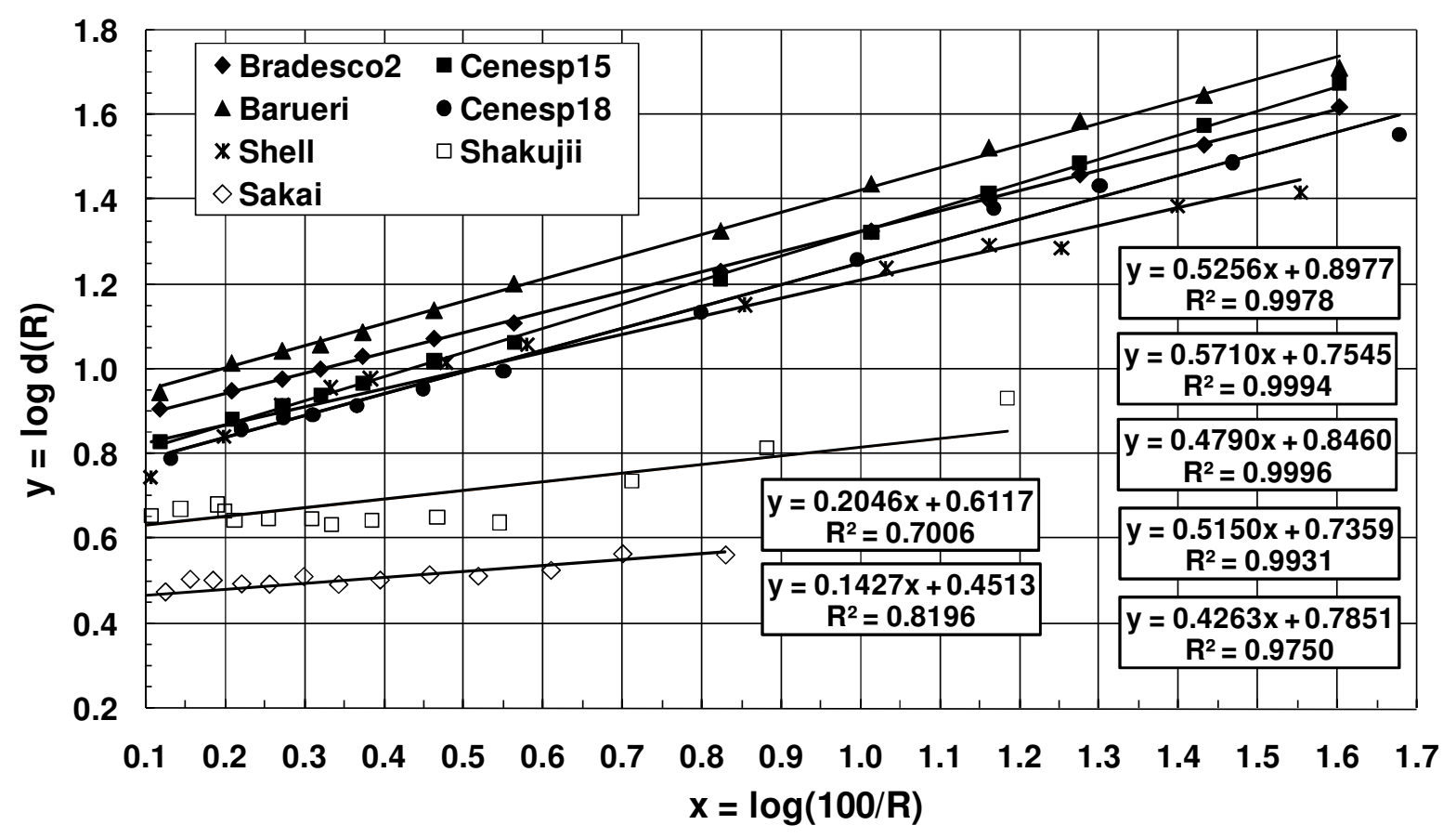

Fig. 3. Determination of effective values for the parameters $\left(\log d_{o}\right)$ and $\beta$, displayed in the boxed equations in the same order of the respective curves (data corresponding to ECL links adapted from references [1]-[4]).

\section{COMPARISON BETWEEN CALCULATIONS AND MEASUREMENTS, AND MODEL PREDICTIONS}

Figure 4 displays the calculated and measured cumulative distribution functions of differential rain attenuation (DRA), corresponding to the converging ECL links. In this Figure, calculated results, represented by continuous or dashed lines, should be compared with measurement results, represented by filled squares or triangles. It is assumed that $A_{o}>0$ and Figure 4 displays both sets of results for $\operatorname{Pr}\left\{a_{1}-a_{2}>A_{o}\right\}$ and $\operatorname{Pr}\left\{a_{2}-a_{1}>A_{o}\right\}$. Results for the converging RIS links are displayed in Figure 5, using the same conventions. 

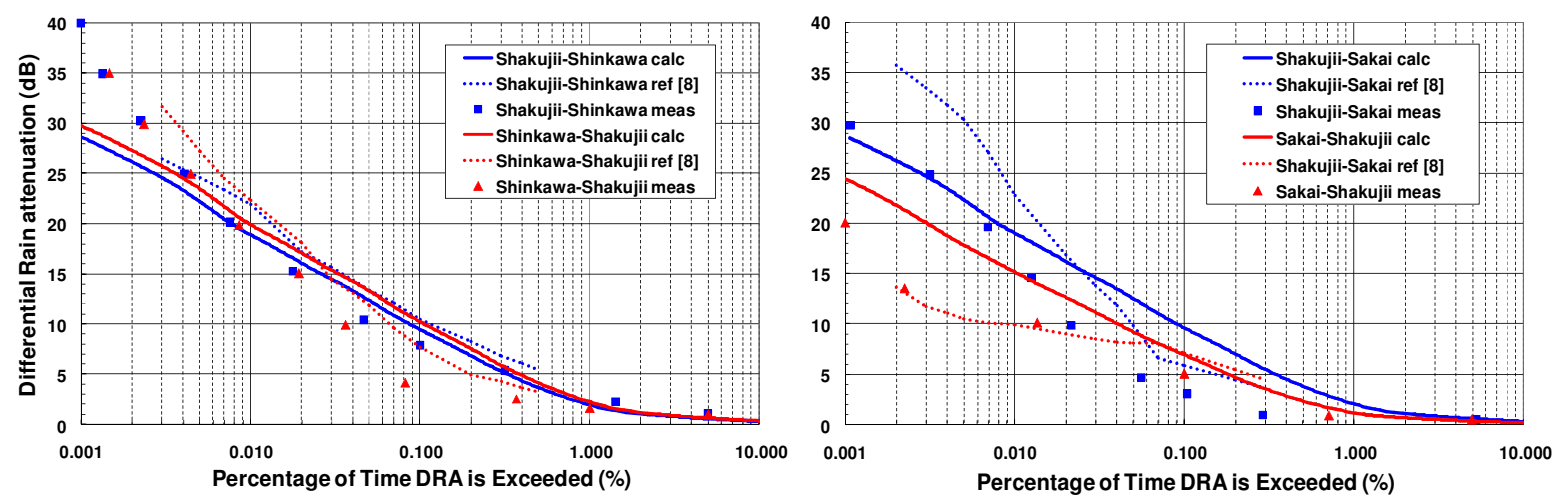

Fig. 4. Calculated (continuous and dashed curves) and measured (filled symbols) cumulative distribution functions for the differential rain attenuation between the converging ECL links described in Section III (adapted from reference [4]).
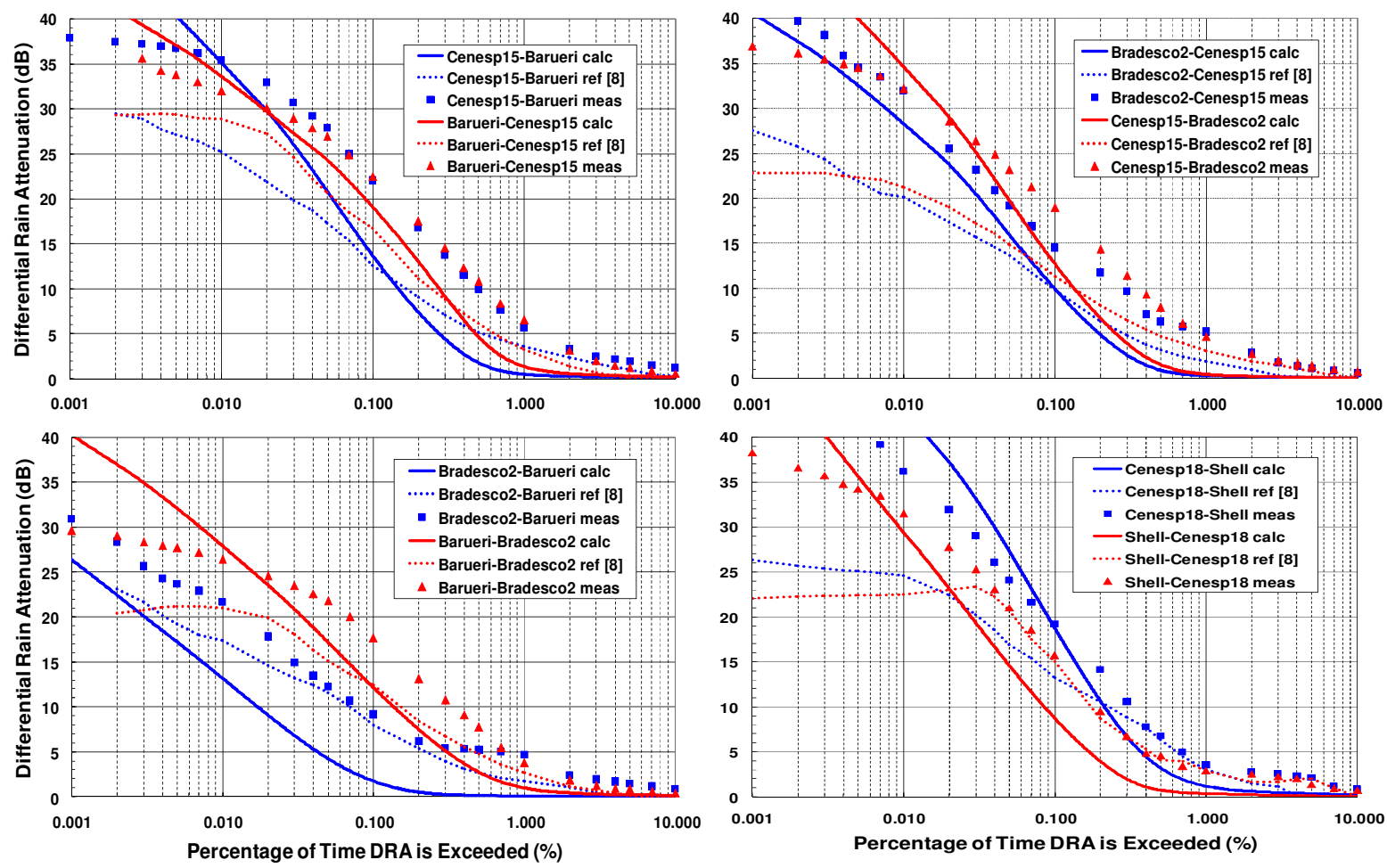

Fig. 5. Calculated (continuous and dashed curves) and measured (filled symbols) cumulative distribution functions for the differential rain attenuation between the converging RIS links described in Section III.

Both Figures also show the results from the prediction model proposed by Garcia et al. [8], which estimates the cumulative distribution function of the differential rain attenuation through

$$
A_{\text {diff }, 12}(p)=\left[a_{1}(p)-0.34 a_{2}(p)\right]\left(\theta^{0.23}+0.004\left|D_{1}-D_{2}\right|^{2.25}\right) f^{-0.4}
$$

In equation (9), $A_{\text {diff, }, 2}(p)$ and $a_{i}(p)(i=1,2)$ are the differential rain attenuation between links 1 and 2 , the rain attenuation in link $i(i=1,2)$ corresponding to the percentage level $p$. The angle $\theta$ between the links should be expressed in radians, the path lengths $D_{i}(i=1,2)$ in kilometers, and the frequency in 
Gigahertz. This model is particularly attractive when the cumulative distribution functions of the rain attenuation on both links are immediately available.

The agreement between calculations and measurements displayed in Figures 4 and 5, depending on the tested case, ranges from good to poor. For example, it is good for both models in the cases of the Shakujii-Shinkawa data of Figure 4 for percentages of time greater than $0.004 \%$. On the other hand, it is poor in the case of the Bradesco2-Barueri data, particularly for the proposed model and percentages of time between $0.01 \%$ and $0.1 \%$, and for the model of reference [8] in the cases displayed in the right panels of Figure 5, for percentages of time less than $0.02 \%$.

Figure 5 displays several examples of slow variations of the measured differential rain attenuation as the time percentage decreases below $0.01 \%$. This is due to link design and technical characteristics of the receivers, which reach the reception threshold for attenuations near $37 \mathrm{~dB}$. Therefore, only data from Figures 4 and 5 corresponding to time percentages above $0.005 \%$ were used to determine the average value and the standard deviation of the error $\left\{D R A_{\text {meas }}(p)-D R A_{\text {calc }}(p)\right\}$, where $p$ represents the common percentage of time. Two percentage intervals were used in the calculations: (i) [0.005\%, $10.0 \%$, which covers most of the available data, emphasizing large probabilities and low DRA values; and (ii) $[0.005 \%, 0.03 \%]$, which emphasizes the exceedance probabilities of interest for potential applications of the proposed model. The average values and the standard deviations of the errors for the two models are presented in Table II for each percentage interval, as well as the respective numbers of samples available for the corresponding calculations. The first two lines consider all the pertinent data in Figures 4 and 5, including the relatively long links converging at the RIS station. On the other hand, the last two lines consider only the shorter links converging at the ECL station. The level of agreement observed in Table II is not uncommon in modeling efforts of rain effects on radio links [24],[25]. It is seen that the average values and the standard deviations of the errors resulting from the extended Misme and Fimbel model are generally less than the corresponding figures provided by that of reference [8]. However, the parameters of the latter model were estimated with basis on nineteen Brazilian links, and most of the ones not converging at the RIS station are considerably shorter than those characterized in the last four lines of Table I. Indeed, nine of the links are shorter than $2.0 \mathrm{~km}$ and twelve of them are shorter than $4.5 \mathrm{~km}$. Additionally, expression (9) is applied here to a data set which differs from the original one. These facts may explain why Garcia at al. [8] reported average values and standard deviations of the errors which are substantially less than the ones displayed in Table II.

It should also be stressed that no attempts have been made at minimizing the observed errors through the application of parameter optimization techniques directly to the data in Figures 4 and 5. This could have been done and would certainly improve the observed agreement. However, this procedure has not been used, since it would be in contradiction with the essential assumptions and methodology leading to the original Misme and Fimbel model and its present extension, which were meant to be upheld. Additional possible factors explaining the observed differences between 
calculated and measured distributions are the non-uniformity of the rainfall rate inside the cell, the effects of multiple cells on differential rain attenuation, and errors due to the estimation of $d_{o}$ and $\beta$ through the alternative approximate methodology described in Section III.

TABLE II. AVERAGE AND STANDARD DEVIATION OF ERRORS BETWEEN MEASUREMENT AND PREDICTION RESULTS

\begin{tabular}{ccccccc}
\hline Links & $\begin{array}{c}\text { Percentage } \\
\text { Interval }\end{array}$ & $\begin{array}{c}\text { Number of } \\
\text { Samples }\end{array}$ & \multicolumn{2}{c}{ Proposed Model } & \multicolumn{2}{c}{ Model of Reference [8] } \\
& & & Average & Std. Dev. & Average & Std. Dev. \\
\hline ALL & {$[0.005,10.0]$} & $192 ; 173$ & $2.21 \mathrm{~dB}$ & $3.72 \mathrm{~dB}$ & $3.65 \mathrm{~dB}$ & $4.39 \mathrm{~dB}$ \\
ALL & {$[0.005,0.03]$} & $47 ; 48$ & $0.02 \mathrm{~dB}$ & $4.12 \mathrm{~dB}$ & $6.29 \mathrm{~dB}$ & $5.79 \mathrm{~dB}$ \\
ECL & {$[0.005,10.0]$} & $25 ; 16$ & $-2.60 \mathrm{~dB}$ & $2.53 \mathrm{~dB}$ & $-2.75 \mathrm{~dB}$ & $5.27 \mathrm{~dB}$ \\
ECL & {$[0.005,0.03]$} & $8 ; 8$ & $-2.75 \mathrm{~dB}$ & $1.90 \mathrm{~dB}$ & $-2.47 \mathrm{~dB}$ & $6.44 \mathrm{~dB}$ \\
\hline
\end{tabular}

Next, the extended Misme and Fimbel model was used to determine the cumulative distributions of the differential rain attenuation between vertically-polarized 15-GHz links located in the São Paulo region (that is, assuming $d_{o}=6.80 \mathrm{~km}$ and $\beta=0.52$ ) with path lengths $D_{l}=D_{2}=10 \mathrm{~km}$, for angles varying between $5^{\circ}$ and $50^{\circ}$ with $5^{\circ}$ increments and between $50^{\circ}$ and $180^{\circ}$ with $10^{\circ}$ increments. The resulting distributions for increasing angles are displayed in Figure 6 from left to right. Fast variations in the curves as a function of the angle between the links are initially observed, for angles less than approximately $60^{\circ}$. However, the same variation became extremely slow beyond this threshold. Note that, for any fixed time percentage, the differential rain attenuation initially increases and gradually tends to a constant value as the angle between the links increases [4].

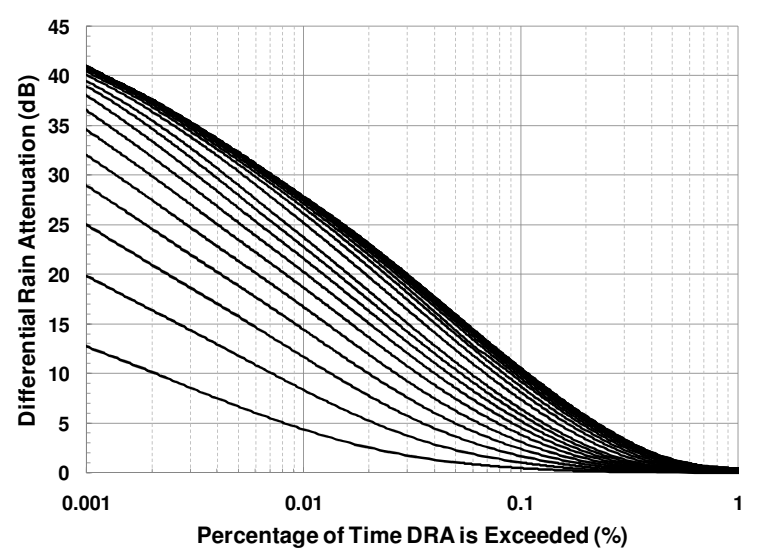

Fig. 6. Cumulative distribution functions of the differential rain attenuation presented from left to right for angles varying between $5^{\circ}$ and $50^{\circ}$ with $5^{\circ}$ increments and between $50^{\circ}$ and $180^{\circ}$ with $10^{\circ}$ increments, assuming vertically-polarized $15-$

$\mathrm{GHz}$ links located in the São Paulo region $\left(d_{o}=6.80 \mathrm{~km}\right.$ and $\left.\beta=0.52\right)$ with fixed path lengths $D_{l}=D_{2}=10 \mathrm{~km}$.

The present model was also used to analyze the effects of the increase of the path length of one link on the cumulative distributions of the differential rain attenuation. This study was performed for conditions of the São Paulo region and vertically-polarized 15-GHz links, fixing the path lengths $D_{l}=$ $10 \mathrm{~km}$ and $\theta=15^{\circ}$, while the variable path length $\mathrm{D}_{2}$ increased from $3 \mathrm{~km}$ to $15 \mathrm{~km}$ in $1-\mathrm{km}$ increments. The resulting distributions for increasing values of $D_{2}$ are displayed in Figure 7 from right 
to left. Fast variations in the curves as a function of $D_{2}$ are initially observed, for path lengths less than $10 \mathrm{~km}$. No variations were observed beyond this threshold. For any fixed time percentage, the differential rain attenuation initially decreases and gradually tends to a constant value as the path length $D_{2}$ increases.

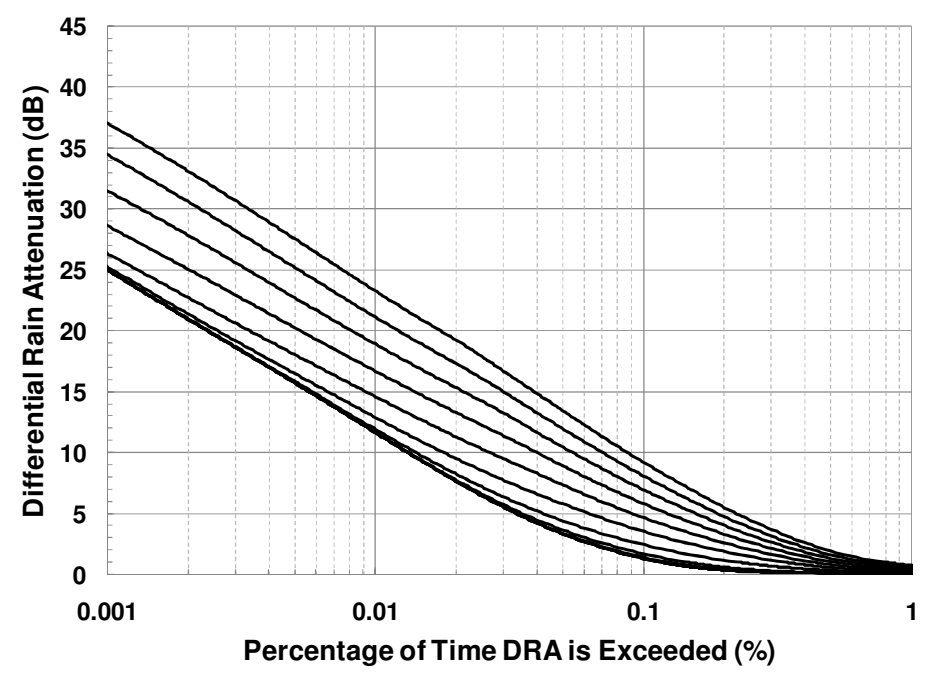

Fig. 7. Cumulative distribution functions of the differential rain attenuation presented from right to left for increasing values of the path length $D_{2}$ (from $3 \mathrm{~km}$ to $15 \mathrm{~km}$ in 1-km increments), assuming vertically-polarized $15-\mathrm{GHz}$ links located in the São Paulo region $\left(d_{o}=6.80 \mathrm{~km}\right.$ and $\left.\beta=0.52\right)$ with fixed path length $D_{l}=10 \mathrm{~km}$ and angle $\theta=15^{\circ}$.

The above predictions can be explained as follows. Let a cell of rainfall rate $\mathrm{R}$ cause attenuations $A_{o l}$ and $A_{o 2}$ on the two converging links $A B$ and $A C$ of Figure 1, respectively. These values define the geometric loci sketched in the same Figure, as already discussed. If the center of the rain cell is located inside the polygon $V_{11} V_{21} V_{31} I_{1} V_{12} I_{2}$, then $a_{1}>A_{o l}, a_{2}<A_{o 2}$, and $a_{1}-a_{2}>A_{o 1}-A_{o 2}=A_{o}$. The dotted area $S\left(A_{o l}, R\right)-S_{\cap}\left(A_{o l}, A_{o 2}, R\right)$ of this polygon, which directly contributes to $\operatorname{Pr}\left\{a_{1}-a_{2}>A_{o}\right\}$, initially increases very rapidly when the angle $\theta$ increases from zero. Beyond a certain threshold which depends on the parameters $A_{o l}, A_{o 2}$, and $R$, this increase becomes very slow. This explains the dependence of the cumulative distributions of the differential rain attenuation on the angle between the two converging links with fixed path lengths, observed in Figure 6. On the other hand, the same area initially decreases when the path length $D_{2}$ of link $A C$ increases from zero, while the path length $D_{1}$ of link $A B$ and the angle $\theta$ are kept unchanged. Another threshold, also dependent on $A_{o l}, A_{o 2}$, and $R$, will be reached beyond which the far end of the geometric locus associated with link $A C$ will be located outside the geometric locus associated with link $A B$. Beyond this threshold, the area of the polygon $V_{1 I} V_{2 I} V_{3 I} I_{1} V_{12} I_{2}$, dotted in Figure 1, will no longer change. This explains the observations in Figure 7. It should be recognized that the present arguments have been presented for fixed values of $A_{o 1}, A_{o 2}$, and $R$, and that there are infinitely many combinations of these parameters which lead to a pre-specified value of $A_{o}$. However, these arguments can be repeated for all combinations of these parameters yielding $A_{o}$, confirming the above justifications for the observed dependences on the angle 
between the links and the path length. Note that prediction results entirely consistent with those in Figure 6 were previously presented by Morita and Higuti [4] without explanations.

\section{DEPENDENCE OF THE AVAILABILITY OF A LINK ON THE DIFFERENTIAL RAIN ATTENUATION}

This section analyzes two procedures to determine the cumulative distribution function of the ratio $C / I(\mathrm{~dB})$ between the desired and interference powers at the same receiver, assuming the existence of only two vertically-polarized 8-km converging links operating at the frequency of $15 \mathrm{GHz}$ under the climatic conditions of the São Paulo region $\left(d_{o}=6.80 \mathrm{~km}\right.$ and $\left.\beta=0.52\right)$. The first procedure is conservative: it considers the rain attenuation $A_{d}(\mathrm{~dB})$ on the desired link and neglects the attenuation $A_{i}(\mathrm{~dB})$ on the interfering link, in such a way that the ratio $C / I$ is estimated using the simple expression $C / I=(C / I)_{o^{-}} A_{d}$. The second procedure is realistic and considers both attenuations. In this case, the ratio $C / I$ and the differential rain attenuation are related through $C / I=(C / I)_{o^{-}}\left(A_{d}-A_{i}\right)=(C / I)_{o}-A_{d i f f}$. In both expressions, $(C / I)_{o}=-g_{r d}(\theta)$ represents the nominal value of the ratio $C / I$, assuming clear-sky conditions, and $g_{r d}(\theta)$ is the discrimination $(\mathrm{dB})$ of the receiving (interfered with) antenna for the angle $\theta$ between the links, provided by the corresponding normalized radiation pattern. The $(C / I)_{o}$ values of $50 \mathrm{~dB}, 45 \mathrm{~dB}$, and $40 \mathrm{~dB}$, assumed in the present study for the angles of $50^{\circ}, 20^{\circ}, 12^{\circ}$, respectively, are inferior to the worst-case values provided by Recommendation ITU-R F.699-7 [26] for 6 -ft $(1.8 \mathrm{~m})$ antennas operating at $15 \mathrm{GHz}$ and consider equal equipment on both links. Both $A_{d}$ and $A_{\text {diff }}$ are random variable, and their cumulative distribution functions were determined by the models described in section II, considering the above angles, when applicable. From these results and the above expressions, the cumulative distribution functions of the random variable $C / I$ were obtained for the two procedures and the three values of $(C / I)_{o}$. Figure 8 displays the results for the conservative procedure using dashed lines with hollow symbols and those for the realistic procedure using continuous lines with filled symbols. Note that a common symbol is adopted for each value of $(C / I)_{o}$. The results indicate that, for $C / I<35 \mathrm{~dB}$, regardless of the value of the ratio $(C / I)_{o}$ (or, equivalently, of the angle between the links), the time percentage that any given level of the ratio $C / I$ is not exceeded is always greater when calculated by the conservative procedure.

To analyze the impact of the above procedures for the estimation of the effects of the differential rain attenuation on the availability of a link and on the efficient use of the electromagnetic spectrum, link-design criteria have to be established. It will be assumed that the thermal noise power level is much less than that of interference and can be neglected. It will also be assumed that a link is unavailable when $C / I \leq 20 \mathrm{~dB}$. As a design objective, it will be required that, due to differential rain attenuation, the link should be available during $99.99 \%$ of the time (that is, it should be unavailable during less than 53 minutes in a year). In other words, to meet this availability objective, the C/I cumulative distribution curve for the link should intercept the black horizontal bar displayed in Figure 8. Note that the curves corresponding to the conservative procedure and to the values of $40 \mathrm{~dB}$ and 45 $\mathrm{dB}$ for the ratio $(\mathrm{C} / \mathrm{I})_{\mathrm{o}}$ do not meet the availability objective. To strictly meet it, the designer using this 
procedure would have to aim at a very high 50-dB attenuation margin for the link and to avoid reusing the same frequency and the same polarization for other converging links making angles less than $50^{\circ}$ with the planned one. Therefore, only seven links converging at the central station would be able to share the same frequency and polarization. On the other hand, based on the realistic procedure, it would be possible to design the same link with a $40-\mathrm{dB}$ attenuation margin and to reuse the same frequency and polarization for links making angles of $12^{\circ}$ with the planned one. In this case, 31 links converging at the central station would be able to share the same frequency and polarization. Note that the present analysis is based on a best-case scenario, which assumes only one interfering link. Additional interference from other links would degrade the C/I curves in Figure 8, moving their intersections with the availability objective bar to the right. This may be critical in terms of the conservative procedure, but Figure 8 indicates that designs based on the realistic procedure would support additional degradation.

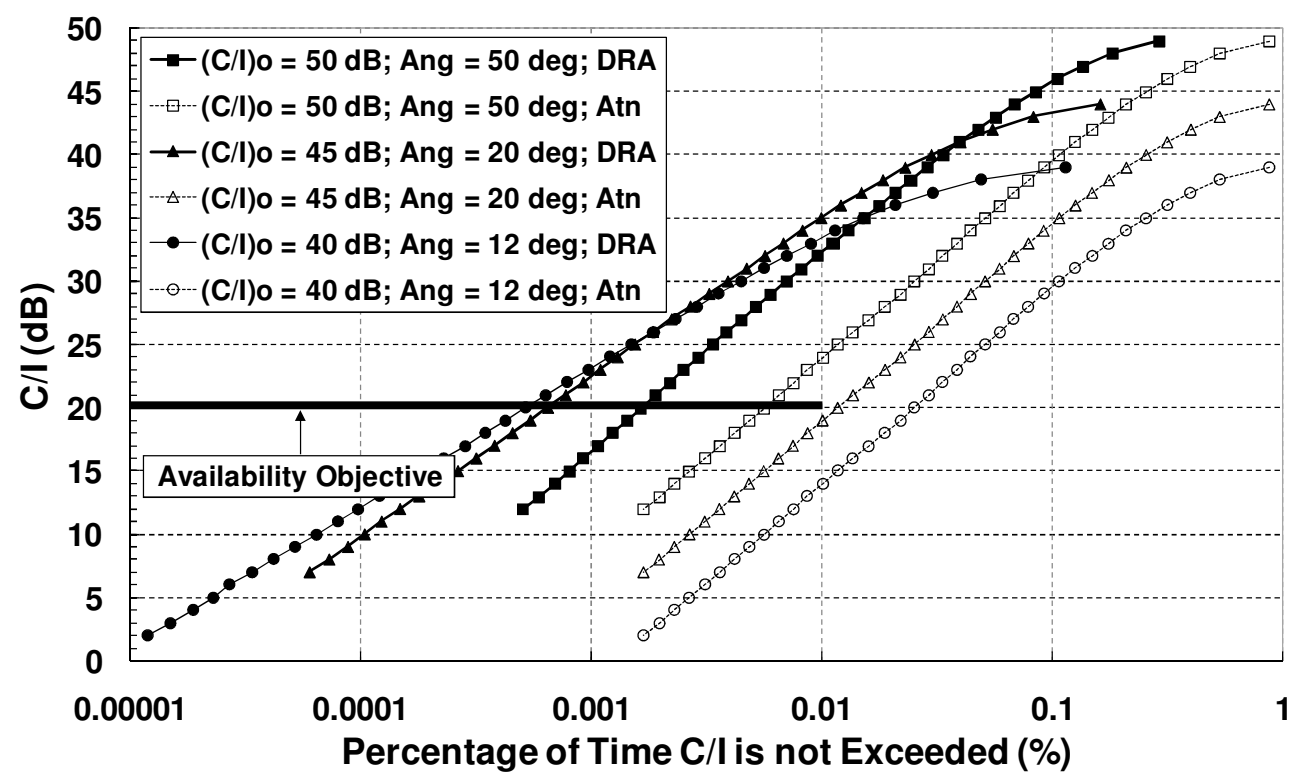

Fig. 8. Cumulative distribution functions of the ratio $\mathrm{C} / \mathrm{I}$ for the conservative (dashed lines with hollow symbols) and realistic (continuous lines with filled symbols) procedures, and three values of the ratio $(\mathrm{C} / \mathrm{I})_{\mathrm{o}}$ (or, equivalently, three angle between the converging links), as well as the availability objective adopted in link design

\section{CONCLUSION}

This work presented a distribution-free method that extends the Misme and Fimbel model to determine the cumulative distribution function of the differential rain attenuation between two converging links in co-channel operation at frequencies greater than $10 \mathrm{GHz}$.

Model predictions were compared with results from measurements performed by Morita et al. [1][4],and by the Radio Propagation Group at CETUC/PUC-Rio [7],[8], as well as with those from another model [8]. These comparisons justify the introduction of the present model. However, additional tests involving other models and additional experimental data are necessary to a better understanding of the present line of study. 
Observed differences between the predictions from the proposed model and the experimental results could be attributed to several factors: the existence of prevailing directions of rain structures in the region, different cell shapes, non-uniformity of the rainfall rate inside the cell, and the effects of multiple cells on differential attenuation. A very important source of errors is the estimation of the parameters $d_{o}$ and $\beta$ by the methodology described in Section III. Possibly different distributions for convective and stratiform rain may not be adequately considered by the model. The inclusion of any of these effects in more refined models would be a challenging task, open to future contributions to this area of study.

Next, the extended Misme and Fimbel model was used to determine the effects of the increase of the angle between the links and of the path length of one link on the cumulative distribution of the differential rain attenuation. For a fixed time percentage, a fast increase of the differential rain attenuation with the angle was initially observed. However, the increase became very slow beyond a threshold. On the other hand, the initially fast decrease the differential rain attenuation with the path length of the interfering link ceased to occur beyond another threshold. Explanations for these observations were provided in terms of the geometric loci associated with attenuation levels on the two links. One of the positive features of prediction methods based on fundamental principles such as the extended Misme and Fimbel model is that they may provide physical explanations and better understanding of the effects of different input parameters on the observed variables. This may provide useful guidance to designers and researchers in their respective works.

Two procedures to determine the cumulative distribution function of the ratio $\mathrm{C} / \mathrm{I}(\mathrm{dB})$ between the desired and interference powers at the same receiving antenna were also analyzed, for different angular discrimination values. The first procedure is conservative: considers the rain attenuation on the desired link and neglects the attenuation on the interfering link. The second procedure is more realistic and considers both attenuations. To analyze the impact of the above procedures for the estimation of the effects of the differential rain attenuation on the availability of a link and on the efficient use of the electromagnetic spectrum, a link-design objective was established. To strictly meet the objective, the designer using the conservative procedure would have to aim at a 50-dB attenuation margin for the link and to avoid reusing the same frequency and the same polarization for other converging links making angles less than $50^{\circ}$ with the planned one. However, based on the more realistic procedure, it would be possible to design the same link with a 40-dB attenuation margin and to reuse the same frequency and polarization for links making angles of $12^{\circ}$ with the planned one. That is, these results indicate that the more realistic procedure could provide more economic link designs and more efficient use of the spectrum. Therefore, it would be interesting to confirm these calculations by the analysis of additional configurations and by appropriate measurements.

Finally, it should be observed that the application of the present model to converging satellite links is possible. For paths with moderate to high elevations, the horizontal projections of these links would 
be relatively short and the assumption that attenuation is caused by a single rain cell is expected to hold. Such an application may be the object of a future report.

\section{ACKNOWLEDGMENT}

The authors thank Dr. Rodolfo S. L. de Souza (Instituto Nacional de Metrologia, Normalização e Qualidade Industrial, Rio de Janeiro, Brazil) and Prof. N. A. Pérez García (Universidad de los Andes, Mérida, Venezuela) for providing and processing the Cenesp18-RIS/Shell-RIS data, respectively. They also thank the reviewers for their detailed and insightful comments and constructive suggestions. Their valuable contributions helped the authors to produce an improved paper.

\section{REFERENCES}

[1] M. Shimba, K. Morita, and A. Akeyama, "Radio propagation characteristics due to rain at 20-GHz band," IEEE Transactions on Antennas and Propagation, vol. 22, no. 3, pp. 507-509, May 1974.

[2] K. Morita, O. Sasaki, and A. Akeyama, "Differential rain attenuation on adjacent 20-GHz band links," IEEE Transactions on Antennas and Propagation, vol. 23, no. 2, pp. 300-301, March 1975.

[3] O. Sasaki, K. Morita, and M. Kikushima, "19-GHz rain attenuation characteristics on four tandem links," IEEE Transactions on Antennas and Propagation, vol. 24, no. 2, pp. 216-220, March 1976.

[4] K. Morita and I. Higuti, "Prediction of differential rain attenuation on adjacent microwave and millimeter wave links," Review of the Electrical Communication Laboratories, vol. 25, no. 1-2, pp. 96-103, January-February 1977.

[5] J. D. Kanellopoulos and S. G. Koukoulas, "Analysis of the rain outage performance of route diversity systems," Radio Science, vol. 22, no. 4, pp. 549-565, July-August 1987.

[6] A. D. Panagopoulos and J. D. Kanellopoulos, "Statistics of differential rain attenuation on converging terrestrial propagation paths," IEEE Transactions on Antennas and Propagation, vol. 51, no, 9, doi: 10.1109/TAP.2003.816643, pp. 2514-2517, September 2003.

[7] L. A. R. da Silva Mello, E. Costa, and R. S. L. Souza, "Rain attenuation measurements at 15 and 18 GHz," Electronics Letters, vol. 38, no. 4, doi: 10.1049/el:20020105, pp. 197-198, February 2002.

[8] N. A. Pérez García, L. A. R da Silva Mello, and M. S. Pontes, "Measurements and prediction of differential rain attenuation in convergent links," Electronics Letters, vol. 41, no. 17, doi: 10.1049/el:20052223, pp. 942-943, August 2005.

[9] F. J. A. Andrade, L. A. R. da Silva Mello, M. S. Pontes, and M. E. C. Rodrigues, Statistical Modeling of Rain Attenuation in Tropical Terrestrial Links, Journal of Microwaves, Optoelectronics and Electromagnetic Applications, Vol. 11, no. 2, pp. 296-303, December 2012.

[10] K. S. Paulson, R. J. Watson, and I. S. Usman, "Diversity improvement estimation from rain radar databases using maximum likelihood estimation," IEEE Transactions on Antennas and Propagation, vol. 54, no. 1, doi: 10.1109/TAP.2005.861571, pp. 168174, January 2006.

[11] P. Misme and J. Fimbel, "Détermination théorique et expérimentale de l'affaiblissement par la pluie sur um trajet radioélectrique," Annales des Télécommunications, vol. 30, no. 5-6, pp. 149-158, November-December 1975.

[12] L. Stola, "An approach to compute the performance of route diversity systems at frequencies above $10 \mathrm{GHz}$," CSELT Rapporti Tecnici, vol. IX, no. 6, pp. 617-620, December 1981.

[13] Specific Attenuation Model for Rain for Use in Prediction Methods, ITU-R (2005), Recommendation ITU-R P.838-3.

[14] O. Rue, "An improved method for rain attenuation prediction on terrestrial and Earth-space paths," in Proceedings of the 12 European Microwave Conference, Helsinki, Finland, October 4-6, 1982, pp. 578-583.

[15] A. Papoulis, Probability, Random Variables, and Stochastic Processes. New York: McGraw-Hill, 1991.

[16] J. O'Rourke, Computational Geometry in C. New York: Cambridge University Press, 2005.

[17] J. Goldhirsh and B. Musiani, "Rain cell size statistics derived from radar observations at Wallops island, Virginia," IEEE Transactions on Geoscience and Remote Sensing, vol. 24, no. 6, pp. 947-954, November 1986.

[18] C. Capsoni, F. Fedi, C. Magistroni, A. Paraboni, and A. Pawlina, "Data and theory for a new model of the horizontal structure of rain cells for propagation applications," Radio Science, vol. 22, no. 3, pp. 395-404, May-June 1987.

[19] L. Féral, H. Sauvageot, L. Castanet, and J. Lemorton, "HYCELL - A new hybrid model of the horizontal distribution for propagation studies: 1. Modeling of the rain cell," Radio Science, vol. 38, no. 3, 1056, doi:10.1029/2002RS002802, pp. 22.1-22.20, May-June 2003.

[20] L. Féral, H. Sauvageot, L. Castanet, and J. Lemorton, "HYCELL - A new hybrid model of the horizontal distribution for propagation studies: 2. Statistical modeling of the rain rate field," Radio Science, vol. 38, no. 3, 1057, doi:10.1029/2002RS002803, pp. 23.123.18, May-June 2003.

[21] Q. W. Pan and G. H. Bryant, "Effective rain-cell diameters and rain-column heights in the tropics," Electronics Letters, vol. 30, no. 21, pp. 1800-1802, October 1994.

[22] J. L. R. P. de Cerqueira, M. S. Assis, and L. A. R. da Silva Mello, "Spatial structure of rain in the Amazon region," in Proceedings of the European Conference on Antennas and Propagation, Nice, France, November 06-10, 2006, CD ESA SP-626 347987jc.pdf.

[23] S. K. Sarkar, M. V. S. N. Prasad, H. N. Dutta, B. M. Reddy, and D. N. Rao, "Rain and extent of rain cells over the Indian subcontinent," in Proceedings of the $6^{\text {th }}$ International Conference on Antennas and Propagation, Coventry, UK, April 4-7, 1989, vol. 2, pp. 318-321.

[24] E. Matricciani and C. Riva, "The search for the most reliable long-term rain attenuation CDF of a slant path and the impact on prediction models,"IEEE Transactions on Antennas and Propagation, vol. 53, no. 9, doi: 10.1109/TAP.2005.854539, pp. 3075-3079, September 2005.

[25] N. A. Pérez García and L. A. R. da Silva Mello, "Improved method for prediction of rain attenuation in terrestrial links," Electronics Letters, vol. 40, no. 11, doi: 10.1049/el:20040464, pp. 683-684, May 2004.

[26] Reference radiation patterns for fixed wireless system antennas for use in coordination studies and interference assessment in the frequency range from $100 \mathrm{MHz}$ to about $70 \mathrm{GHz}$, ITU-R (2006), Recommendation ITU-R F.699-7. 